\title{
Conceptual Design of a Communication-based Deep Space Navigation Network
}

\author{
Evan J. Anzalone ${ }^{1}$ and Jason C.H. Chuang ${ }^{2}$ \\ NASA Marshall Space Flight Center, Huntsville, Al, 35801
}

\begin{abstract}
As the need grows for increased autonomy and position knowledge accuracy to support missions beyond Earth orbit, engineers must push and develop more advanced navigation sensors and systems that operate independent of Earth-based analysis and processing. Several spacecraft are approaching this problem using inter-spacecraft radiometric tracking and onboard autonomous optical navigation methods. This paper proposes an alternative implementation to aid in spacecraft position fixing. The proposed method Network-Based Navigation technique takes advantage of the communication data being sent between spacecraft and between spacecraft and ground control to embed navigation information. The navigation system uses these packets to provide navigation estimates to an onboard navigation filter to augment traditional ground-based radiometric tracking techniques. As opposed to using digital signal measurements to capture inherent information of the transmitted signal itself, this method relies on the embedded navigation packet headers to calculate a navigation estimate. This method is heavily dependent on clock accuracy and the initial results show the promising performance of a notional system.
\end{abstract}

$\begin{array}{ll} & \\ D D O R & =\text { Delta Differential One-way Ranging } \\ D S N & =\text { Deep Space Network } \\ E K F & =\text { Extended Kalman Filter } \\ G P S & =\text { Global Positioning System } \\ h_{0} & =\text { white frequency noise } \\ h_{-2} & =\text { frequency random walk } \\ L E O & =\text { Low Earth Orbit } \\ M E R & =\text { Mars Exploration Rover } \\ M R O & =\text { Mars Reconnaissance Orbiter } \\ M S L & =\text { Mars Science Laboratory } \\ N A I F & =\text { Navigation and Ancillary Information Facility } \\ N N A V & =\text { Network-based Navigation } \\ R K & =\text { Runge-Kutta } \\ U H F & =\text { Ultra High Frequency }\end{array}$

\section{Introduction}

$\mathrm{O}$ VER the centuries, humans have developed methods of positioning, which required on some a priori knowledge and the integration of maps and measurement. For ship voyages into uncharted waters, this required tracking the heading of a ship using a compass, and measuring velocity. Using this information, the navigator could determine the location of the vessel. In our modern culture, man has developed artificial measurement systems to aid in position finding. The greatest example of this is the use of Global Navigation Satellite Systems, which have become increasingly prevalent in our lives, with their inclusion in car navigation systems, cellular phones, and airplane systems. Over a small period of time, these systems can easily provide position-fixing accuracy on the order

\footnotetext{
1 Aerospace Engineer, Guidance Navigation and Mission Analysis Branch, MSFC/EV42, Member AIAA, evan.j.anzalone@nasa.gov

${ }^{2} \mathrm{PhD}$, Navigation Lead, Guidance Navigation and Mission Analysis Branch, MSFC/EV42, Member AIAA
} 
of several meters. Such man-made systems require a large infrastructure investment to support operations and maintaining accuracy. For deep space navigation systems, a different set of algorithms and methods have been developed to address the observational environment and vehicle limitations.

For spacecraft traversing deep space, navigation is critical to mission success, enabling ground-based control and data collection, allowing us to return data from the farthest reaches of spacecraft. Deep space navigation is also intrinsically difficult due to the remote distances, limited instrumentation, and complex analysis required. As the capability of onboard instruments is improved, there is strong need for matching high accuracy position information in order to tie back the observations to physical locations and sources being observed. Higher resolution observations therefore directly increase navigation accuracy.

With the development and expansion of communication assets into planetary networks, such as the Mars Network, there exists a great opportunity to integrate a new solar system-wide navigation system. Initial networks, such as Mars Reconnaissance Orbiter (MRO) operating as a communications relay for Mars ground-based assets, show that the capability exists for such a distributed networking system. Research into Delay- and DisruptionTolerant Networking is developing a protocol to be used in such an environment for high-bandwidth reliable communications between assets. The architecture requires the development of a communications backbone consisting of data relays transferring data between regions, such as Mars or Earth. Independent regional networks also exist for local communications. Initial elements in this communication backbone would include Deep Space Network assets, as well as MRO-type satellites. Highly accurate navigation data of any in-space data relays must be known in order to maintain good pointing to enable large amounts of bandwidth. This system can also be modified to allow for in-space navigation, reducing reliance on Deep Space Network (DSN) Earth-based assets, freeing them for communications purposes.

These communication relay satellites can be used as a beacon-based navigation system for craft in transit or in orbit, by attaching a navigation header to each data packet transmitted. To acquire a navigation fix, the travelling craft will search for any beacon or communications transmissions. Once found, it will point towards the source of the signal and determine a pointing vector. Once pointing is attained, the spacecraft will listen for any navigation data packets. These beacon packets will include any command and control data as well as time of transmission and source of transmission. With the combination of a pointing vector, a time difference between assets and the known location of the beacon, the spacecraft can generate a navigation fix. This study will analyze the performance of such a navigation system by considering the architecture as a whole, and determining which capabilities are required in order to achieve savings over traditional methods. Systems under analysis will include transmission power requirements, pointing requirements, satellite sizing effects, and constellation parameters, such as number of assets and celestial position to quantify the capabilities of such a system.

This research will focus on the development of physics based modeling to capture detailed performance at the spacecraft and network levels, comparing performance to other methods, such as DSN radiometric tracking and optical navigation. This works focuses on the design and analysis of a new navigation method that integrates with communications signals to provide high accuracy position-fixing. Additionally, this work will focus on performance of the network-based navigation algorithms during cruise and planetary orbit to serve as test and validation methods for the navigation technique as an augmentation technique to current navigation practices.

\section{Navigation and Communication Evolution}

There are multiple methods that have been developed to generation deep space navigation solutions and perform communication with probes, rovers, and orbiters. These will be briefly discussed below, to give the reader a quick overview of the state of the field.

\section{A. Navigation Techniques and Current Research}

A prerequisite to any space mission is a means of tracking and providing navigation information to the control authority, whether that is ground operations or autonomous software onboard the spacecraft. There is a range of navigation techniques in use for spacecraft in deep space. Traditional techniques involve optical navigation ${ }^{1}$, in which the bearing and range (or multiple bearings) to a celestial object(s) are combined with known ephemeris information to calculate a position fix. The application of this method is constrained by the optical properties of the observation instrument. Additionally, there is a requirement for ground support to analyze the optical images and compute the navigation fix. Research into Autonav ${ }^{2}$ moves this analysis onboard the spacecraft, at the cost of a large a priori knowledge requirement and increased mission planning to tell the spacecraft when to point and where in 
order to capture observations for a navigation fix. In addition to instrument properties, this capability is also limited by knowledge of the observed object's ephemeris.

Another method is to use radio waves to determine range and radial velocity (or range rate) ${ }^{3}$. The range is calculated by measuring the time it takes a tone to travel to the spacecraft and return to the ground (two-way ranging) and radial velocity is measured by capturing the Doppler shift inherent in the received signal (of an initially known frequency). The angular position in the sky is captured by antenna tracking techniques which utilize specialized hardware and software to analyze the power of the received signal. This measured angular position can be improved by Delta Differential One-way Ranging (DDOR) techniques, which utilize multiple antennas and observations of a known source to capture errors, to the order of nanoradians. This process is very sophisticated, requires a large amount of ground hardware and analytical support. Research into software-defined radios has developed the Electra instrument, which can perform in-orbit radiometric ranging and Doppler tracking. Currently this is only implemented on UHF radios, which limit the range and applicability of the solution.

Additional methods utilize dead reckoning and measurements of the intrinsic state through highly accurate inertial navigation units. These instruments, though, have some error and due to the integration process, these will always increase over time. The use of X-ray navigation is also being research for deep space missions ${ }^{4,5}{ }^{5}$.This technique uses observations of high energy pulsars with highly detailed pulse arrival models, to capture the difference in arrival between that measured and at a reference location. This information can be used to compute a navigation fix, but requires large detectors with a long integration time to capture enough data that can then processed through complex folding algorithms to determine the pulse arrival time and phase.

\section{B. Communication Techniques and Current Research}

As more spacecraft are launched with greater reliability and improved scientific instruments return higher resolution data of a greater quantity, the communication system bandwidth begins to become constrained. In order to meet the growing data requirements from individual spacecraft and increases in network traffic to deep space, the current communication system must grow to meet this increasing demand. There are several ways to address this. A simple approach is to develop data compression schemes to reduce total data transmitted. But there is a limit to the effectiveness of this, and current methods are fairly mature. A more productive method is switching to higher frequency standards in order to operate at increased data rates. This has been implemented with the switch from early S-Band to X-band and currently to Ka-Band communication standards which allow for increased bandwidth. These higher frequencies required advancements in radio processing, receivers, and transmitters both on the ground and in space.

To achieve higher data rates by frequency alone requires a fundamental shift in the underlying physics. This change is from transmission and reception of electromagnetic waves to generation and detection of photons from a laser source. This method is known as optical communication and has been in development as an alternative to radio communication $^{7,8}$. Much work has been done in this area to improve data rates, transmission power, and detection sensors. Recently, the NASA Office of Chief Technologist Technology Demonstration Mission ${ }^{9}$ program funded a proposal to fly a next generation optical communications system. The drawback to such a well performing system is the requirement of new satellite subsystems, as well as ground infrastructure.

A different approach is to build out a network architecture into the solar system, breaking down the network into a series of local networks and utilizing relays with high bandwidth data trunks ${ }^{10}$. An example of this is the Mars Reconnaissance Observer (MRO). This spacecraft contains a very powerful transmission capability utilizing Kaband and X-band transmitters. One of the satellite's missions is to operate as a relay for the Mars Exploration Rovers (MER) and other Mars surface assets. Additional studies ${ }^{11}$ have been conducted on expanding the assets in Martian orbit into a Mars Network for data communications amongst assets and to Earth and using these sources for navigation ${ }^{12}$. As more surface assets are utilized, the need for a dedicated data trunk between local orbit and Earth increases. The use of relays for local communications and rovers is well proven and continues to be an aspect of deep space communication architecture.

This can be seen in the current plans for the development and growth of the current space communications architecture. The NASA Space Communications Architecture Working Group ${ }^{13}$ developed a set of heuristics to develop a growth plan to meet data return requirements and frequency constraints in the immediate timeframe. The resulting architecture displays a combination of direct satellite communications combined with data relays, as they become cost-effective, to handle increasing data traffic. The architecture thus provides pathways for additional data relays as part of an evolving infrastructure.

The concept of data relays is key to the InterPlanetary Internet (IPN) concept ${ }^{14}$. The IPN studies focused on an Internet-like approach to networking for space systems. In order to achieve networking in space, a new data transfer 
protocol is required in order to take into the long light travel times between nodes. Using standard TCP/IP data protocols, a packet would timeout very quickly. For example most ground system network protocols assume instantaneous data transfer capability. But this amount of time is barely long enough to enable communication with the moon ${ }^{15}$.This inspired the creation of Delay and Disruption-Tolerant Networking (DTN) protocols, such as the bundle protocol and Licklider Transmission Protocol(LTP).

The combination of LTP and DTN protocols enable a very efficient method of transferring data at large distances, increasing data throughput by minimizing redundant transmissions. These become especially useful as the deep space communication network continues to grow and additional surface and space assets are integrated into the network. There has been ongoing development and flight experience in deep space, such as EPOXI ${ }^{16}$ and the Deep Impact Network Experiments ${ }^{17}$. The Disaster Monitoring Constellation satellite sensor network utilized the bundle protocol to download images from orbit ${ }^{18}$. These protocols provide a standard that can be implemented across transmission mediums to effectively transfer data. The inherent structure of the bundles (and breaking down the data sets into transmittable chunks) provides a great potential for the standard integration of additional information to support space operations, such as dynamic routing network information or navigation aids.

\section{Network-Based Navigation Concept}

This system is envisioned to take advantage of the increasing bandwidth of developing communications and increasing number of in-space data relays to provide an autonomous navigation capability. This uses the communications signals as observables in an online filter to provide updates to the spacecrafts estimated position. By embedding the required navigation directly into the command and control packets, navigation fixes are generated as the spacecraft is processing the data. The data required can also be programmed to be a standard part of the navigation messages and will not require any additional measurements, but simply integrating measurements that are already being used and transmitted them to the spacecraft in real-time. This reduces any reliance on human operators to process the data and the long passes currently required to generate sufficient data in order to perform high accuracy orbit determination on the ground. An initial implementation of this method is to have it serve as an augmentation to traditional ground-based radiometric tracking. These measurements (in addition to GPS and other measurements in LEO prior to Earth-orbit ejection) will still be used to initialize the spacecraft's state to high accuracy. Additionally, the navigation packets would initially serve as an augmentation to traditional methods, with its primary benefit to maintain spacecraft position knowledge accuracy, while reducing the need for long tracking passes during cruise (where downlink data requirements are not as pressing) reducing operational cost.

Network-based navigation seeks to take advantage of a growing communication infrastructure to integrate a positioning capability into the individual data packets. This enhancement will allow for increased navigation with higher precision and greater frequency. The core of the method is utilizing all assets in the infrastructure to perform tracking duties to each other. Whenever two spacecraft are in range to communicate and operations allows for a contact, the two assets attempt a contact, and as part of this contact, the receiving satellite updates its position based on the navigation header received and measured time delay. The primary navigation nodes that will be used for this purpose will be the data relays that form the basis of the expanding complex network. As the individual spacecrafts communicate with each other and provide positioning functionality, the overall navigation accuracy and knowledge of individual position is increased as updated references are propagated throughout the network. 


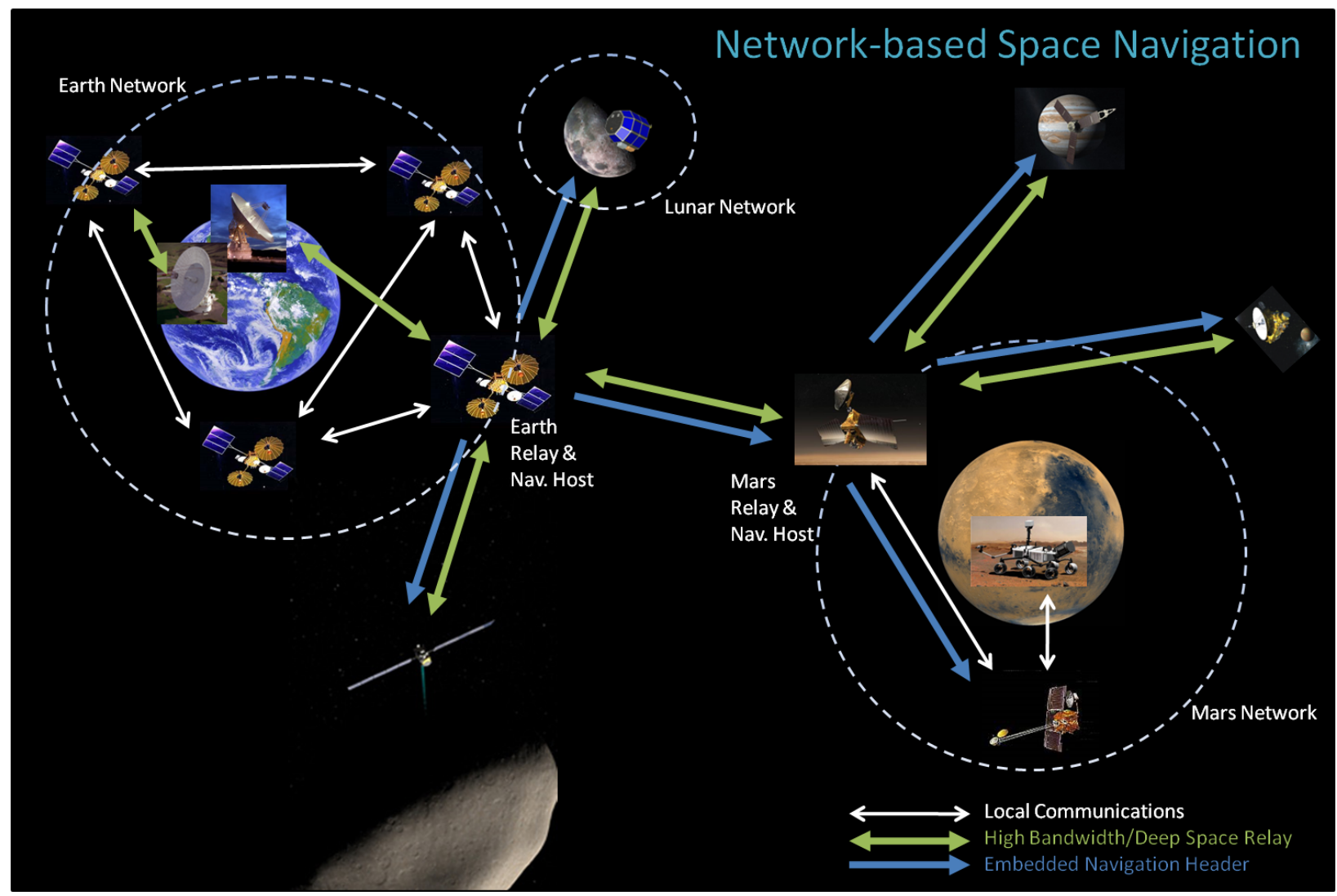

Figure 1. Concept of Operatiosn for a Notional Network-based Navigation Infrastructure

A concept of operations is given in Figure 1. As shown, the growing space communication network is seen as being made up of local planetary networks, such as Lunar, Earth, and Martian. Each network also hosts a high bandwidth-capable relay satellite whose primary purpose is to act as a router in this large network. Additionally a high bandwidth relay-to-ground trunk exists to transfer data to mission control centers. These form the backbone of the interplanetary network, as envisioned both the working group mentioned above and the Interplanetary Internet research efforts. The addition of a Network-based Navigation software solution integrates the capability to process and send the navigation packets amongst hosts. Relay satellites will not only forward data, but will serve as navigation hosts, producing these packets and tracking their own location to high accuracy. As spaceflight transfer from one network to another, and between planets, it is possible to receive navigation packets from multiple relays, thus improving the sources of measurements and including additional degrees of freedom. For trajectories into the outer planets, as signal noise gets higher and higher, and the accuracy of Earth-based ranging begins to increase, the use of navigation hosts deeper into the solar system, closer to the spacecraft, can provide additional resolution and accuracy. This capability depends on the communication ability of the spacecrafts, and will require pointing maneuvers to align high-gain antennas to point not only at Earth hosts but to other spacecraft for transmission in order to receive enough signal power to overcome noise (due to the low power available for transmission and large distances involved).

The initial study will focus on a subset of this proposed method to capture the notional performance of networkbased navigation in a use-case in which it supplements traditional navigation methods. This research will capture the performance of the use of communication-based header integration for cruise navigation. The immediate benefit from integrating this analysis is to reduce the need for expensive DSN passes during cruise, while still maintaining and improving navigational accuracy in positioning. These will be analyzed as measurement updates to state of the art statistical deep space navigation filters. This analysis case will serve as a comparison to the real world data available to capture the method's performance. Cruise navigation performance is ideal for initial implementation due to the simplification of dynamics available and steady orbital trajectory properties. The research will focus on characterizing the errors and uncertainties present affecting deep space navigation and apply filtering techniques to correct. 
Additionally, the size of network infrastructure will be studied to capture the effect of positioning performance during cruise. This will be performed by the introduction of additional data relays into planetary orbits that can be used as navigation as well as communication hubs. The research will focus on analysis of several trajectories of flown missions. This enables calibration and validation to published flight and performance data, as well as ensures the analysis case captures reality well. The trajectories that will be under initial analysis include: Inner Planets Mission (Messenger), Near Planets Mission (MRO or Dawn), Far Planets Mission (Cassini or Juno), and Deep Planet Mission (New Horizons). These cases will serve to show the benefit of using deep space navigation relays as well as allow analysis over a range of planetary missions. Additionally, these will serve as technology benchmarks of spacecraft communication and navigation capability.

This new method is an evolutionary development of navigation systems, applying advanced ranging measurement techniques to deep space navigation. This navigation method implementation represents a continued shift in NASA operations, from missions requiring complex constant ground support to increasingly autonomous vehicles. This increasing reliance on onboard systems relaxes ground operational requirements while also reducing cost and reliance on complex expensive Earth-based measurement techniques.

\section{Analysis Approach and Implementation}

In order to capture the performance of notional network-based navigation architecture, a series of simulations were developed using MATLAB ${ }^{\circledR}$ to perform physics-based analysis. The functions used were developed independently in order to fit within a larger future framework, though future work will use these tools for validation and verification of functional performance. The reference space vehicle under analysis is the in-space trajectory of Mars Science Laboratory. The data used was obtained from the public repository published via the NAIF SPICE database ${ }^{19}$.The analysis tools are integrated with the SPICE/MICE toolkit to interface with the standard trajectory data to use as the truth model for the simulation.

\section{A. Simulation Framework}

The initial design of the simulation tools focused on analyzing the performance of NNAV as an augmentation to traditional deep space navigation methods, particularly high accuracy position and velocity state updates from the ground using DSN tracking, measurement, and orbit determination. Starting from initial conditions, the spacecraft propagated its own estimated state, and measurements were generated at fixed time intervals. For simplicity of analysis, it is assumed that navigation updates occur at fixed intervals with a predefined number of measurements in a batch, with a fixed time between individual observations. At reception of a navigation packet, the spacecraft propagates its state to the current time, processes the measurements, and updates its state and uncertainty. Additionally, the spacecraft clock is modeled in order to capture clock uncertainty and dynamics. The clock stability is captured by the Allan variance parameters $h_{0}$ and $h_{-2}$ with values chosen to match the notional performance of a crystal oscillator ${ }^{20,21}$ modeled similarly to that presented by van Dierendonck ${ }^{22}$.

\section{B. Measurement Models}

Several measurement models were implemented and are possible to be traded in the simulation environment. This design enables understanding the tradeoffs between packet information and defines what data the navigation packet contains. The base measurement of the NNAV system contains the position and time of transmission from a navigation host to a receiving spacecraft. It is assumed that this information is in the same frame as the spacecraft's onboard state propagation. The spacecraft combines this data with measured time of reception to estimate one way travel time similar to a one-way radiometric ranging technique, except instead of tracking carrier-phase the information is explicitly given in the navigation packet. This puts a larger requirement on long-term clock stability and maintaining both short and long term accuracy of time propagation. With the inclusion of pointing information from the transmission host, a vector can be determined between assets, and thus serves as a rough estimate of position (or range if no pointing information is included). Additionally, as more spacecraft become an active part of the network, the capability to correct errors will improve due to the number of potential simultaneous observations.

This measurement can also be used to update the clock state. By comparing the predicted position (or range) with the observed value, this can be used to update the clock bias. The onboard clock bias can be measured by assuming the position is known and given the position and time of transmission, the spacecraft calculate the reception time and compares to it the onboard measured time to update bias. 
One of the advantages of the use of embedded navigation packets is that the spacecraft is able to receive a large quantity of measurement packets to help reduce the inherent noise in the navigation calculations. Additionally, having a series of packets also supports additional measurements. The clock drift rate can be measured by the amount of time measured between packets and the differences between transmission times on subsequent packets. Assuming the clock is fairly stable at the small times between measurements, this can give a good measurement of the speed of the spacecraft clock relative to the true ground clock. Additionally, multiple measurements of range can be used to infer range-rate estimation of the spacecraft, which can be used to update the spacecraft velocity state.

\section{Filter Definition}

In order to track the performance of the measurements to onboard position fixing capability, two separate filtering options were implemented to track spacecraft onboard knowledge. It is assumed that the filter is initialized via traditional high accuracy measurement techniques, reducing the initial error. The spacecraft state is propagated using a Runge-Kutta-Fehlberg $7 / 8^{23}$ algorithm with an relative error tolerance of $10^{-10}$ and an absolute error toleration of $10^{-12}$. Additionally, it is possible to use the built-in ODE45 integrator as an alternative. From initial testing, the two were able to achieve similarly accuracy, but RK78 with a fewer number of iterations required, as such it was chosen to be the standard for this analysis. The integrator is used to propagate states and clock state between measurements, both between batches and individual packets. The filter allows the spacecraft to autonomously track its state. The initial implementation used a batch sequential least squares filter, similar in function to the AutoNAV filtering approach (reference). During a communication pass, the filter would store all of the measurements. Upon completion of the transmission pass, the spacecraft processes these measurements together, performing an orbit determination solution to minimize error between predictions and measured at the time of start of pass. This solution would then be propagated to the next communication pass.

Additionally, an Extended Kalman Filter was implanted to allow for real-time processing and update of the navigation state as each packet is received. Both standard and UD-filters were implemented, though no appreciable difference in numerical accuracy was noticed. The following analysis was performed with the EKF implementation. This filter architecture was chosen due to its real time update capabilities as well as capturing the nonlinear characteristics of the dynamics and measurements and the stochastic nature of the problem.

\section{Initial Results}

In order to capture the notional performance of a packet-based network-based navigation system, the abovedescribed modeling environment was used to perform a series of trades. These results capture the performance of a first order simulation built to get notional results and serve as a jumping-off point for more detailed further analysis. The trades here focus on a performance comparison of multiple measurements techniques and accuracies. The cases modeled are: DSN-level accuracy state updates of position and velocity, packet-based positioning from Earth and MRO, and ranging measurements from Earth and MRO. Additionally, the direct propagation of the initial simulation starting information is included to show a worst-case level of performance and serve as an upper bound. Similarly the DSN measurement cases can be considered to be the current state of the art. All simulations cases also have the initialization error characteristics given in Table 1, assumed to be a high precision DSN-measured state update. The errors of each measurement method are given in Table 2.

The filters developed do not currently model transient accelerations due to effects such as solar radiation pressure, and is captured in the form of the dynamic propagation noise. In order to verify these perturbations are captured and do not greatly affect performance, two sets of simulations are produced. In the first set of cases, a simple propagator is used to calculate the position of MSL as a function of time. The initial state is still captured from the publicly available data (via the NAIF database).

Table 1: Common Error Parameters

\begin{tabular}{|l|l|}
\hline Error Measurement & Value \\
\hline Sigma $\mathrm{x}$ & $1 \mathrm{~km}$ \\
\hline Sigma v & $.1 \mathrm{~km} / \mathrm{s}$ \\
\hline Clock variance, $\mathrm{h}_{0}$ & $1 \mathrm{e}-19$ \\
\hline Clock variance, $\mathrm{h}_{-2}$ & $1 \mathrm{e}-20$ \\
\hline
\end{tabular}

Table 2: Measurement Error Parameters

\begin{tabular}{|l|l|}
\hline Measurement Method & Error \\
\hline
\end{tabular}

American Institute of Aeronautics and Astronautics 


\begin{tabular}{|l|l|}
\hline \multirow{2}{*}{ DSN } & Sigma $\mathrm{r}=1 \mathrm{~km}$ \\
\cline { 2 - 2 } & Sigma $\mathrm{v}=.1 \mathrm{~km}$ \\
\hline Earth/MRO Relative Position & Sigma $\mathrm{r}=100 \mathrm{~km}$ \\
\hline Earth/MRO Range & Sigma $\mathrm{r}=100 \mathrm{~km}$ \\
\hline
\end{tabular}

The second set captures the published design trajectory of MSL, including TCM maneuvers and transient effects. The results will show the capability of the filter to maintain relative accuracy levels in the face of unmodeled forces. Further development of the estimator's dynamics models and onboard propagator will increase the accuracy of the simulation. For both simulations, the available published MRO SPICE telemetry files are used. Due to the delay between file generation and publication, SPICE two-body Mars-centered propagation was used to produce ephemeris files to match the time covering the MSL trajectory. The SPICE ephemeris files are given in Table 3.

Table 3: Ephemeris Files Used

\begin{tabular}{|l|l|}
\hline Asset & SPICE Trajectory File \\
\hline MSL & msl_spk_cruise_od082_v1_tcm2_eph-landed-edl.bsp \\
\hline MRO & mro_psp22.bsp and mro_psp23.bsp \\
\hline Earth & de421.bsp \\
\hline
\end{tabular}

The first set of graphics below shows the performance for a two body propagated orbit for MSL. Each of the cases is described in Table 4. The number of measurements in a batch was obtained through a simple optimization routine. In order to capture performance with the random clock error, several cases were each run at several values. The mean was then taken the family of cases with the same inputs, and the optimal value was selected for these simulations. The $\mathrm{x}$-axis represents time in days from the start of propagation. Using the ephemeris described above, the start of the simulation relates to the calendar data January 12, 2012 02:00:00 UTC. The y-axis shows position and velocity errors in $\mathrm{km}$ and $\mathrm{km} / \mathrm{s}$. Please note that these are plotted on a logarithmic scale. Each case is propagated out to capture 100 measurement batches. The spacecraft clock is also modeled in each case as a stochastic process. The highest accuracy of tracking is produced using position and velocity measurements (of the best precision). Under these conditions, the filter is able to maintain kilometer-level position knowledge. As the measurements contain increased error and less state information, the precision of the filter decreases. The position only observations yield position accuracy on the order of 10s of kilometers and one dimensions ranging yields position knowledge on the order of 1000s of kilometers. For comparison, propagating the initial state (with the given errors) produces errors values greater than $10^{5} \mathrm{~km}$. In terms of velocity error, the direct velocity observation is crucial in maintaining velocity knowledge, but both the ranging and position measurements are able to obtain only one order of magnitude higher in velocity accuracy $\left(10^{-3} \mathrm{~km} / \mathrm{s}\right)$ over time. This is primarily due to the simple dynamics being captured exactly (other than noise) in the estimator's dynamic models.

Table 4: Case Legend and Descriptions

\begin{tabular}{|l|l|l|l|l|}
\hline Case Title & Measurements & $\begin{array}{l}\text { Time between } \\
\text { Measurements }\end{array}$ & $\begin{array}{l}\text { Measurements } \\
\text { in a batch }\end{array}$ & $\begin{array}{l}\text { Time between } \\
\text { individual } \\
\text { measurements }\end{array}$ \\
\hline Simple prop & None & n/a & n/a & n/a \\
\hline Simple DSN daily & Earth-centric position and velocity & 1 day & 1 & n/a \\
\hline Simple DSN weekly & Earth-centric position and velocity & 1 week & 1 & n/a \\
\hline $\begin{array}{l}\text { Simple mroearth daily } \\
4\end{array}$ & $\begin{array}{l}\text { Position relative to Earth and } \\
\text { MRO }\end{array}$ & 1 day & 4 & 15 minutes \\
\hline $\begin{array}{l}\text { Simple mroearth } \\
\text { weekly 4 }\end{array}$ & $\begin{array}{l}\text { Position relative to Earth and } \\
\text { MRO }\end{array}$ & 1 week & 4 & 15 minutes \\
\hline Simple ranging daily 8 & Range from Earth and MRO & 1 day & 8 & 15 minutes \\
\hline $\begin{array}{l}\text { Simple ranging } \\
\text { weekly 4 }\end{array}$ & Range from Earth and MRO & 1 week & 8 & 15 minutes \\
\hline
\end{tabular}




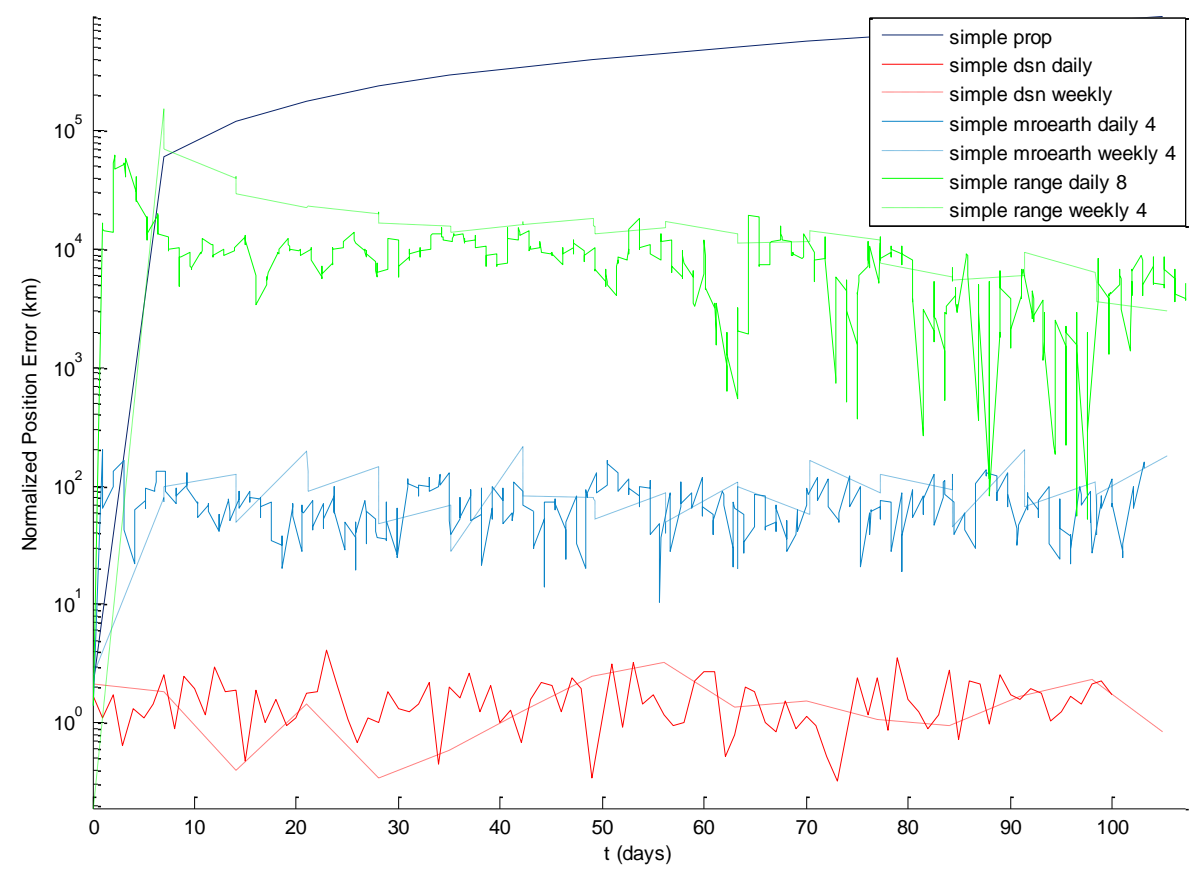

Figure 3: Position Error for MSL Propagated

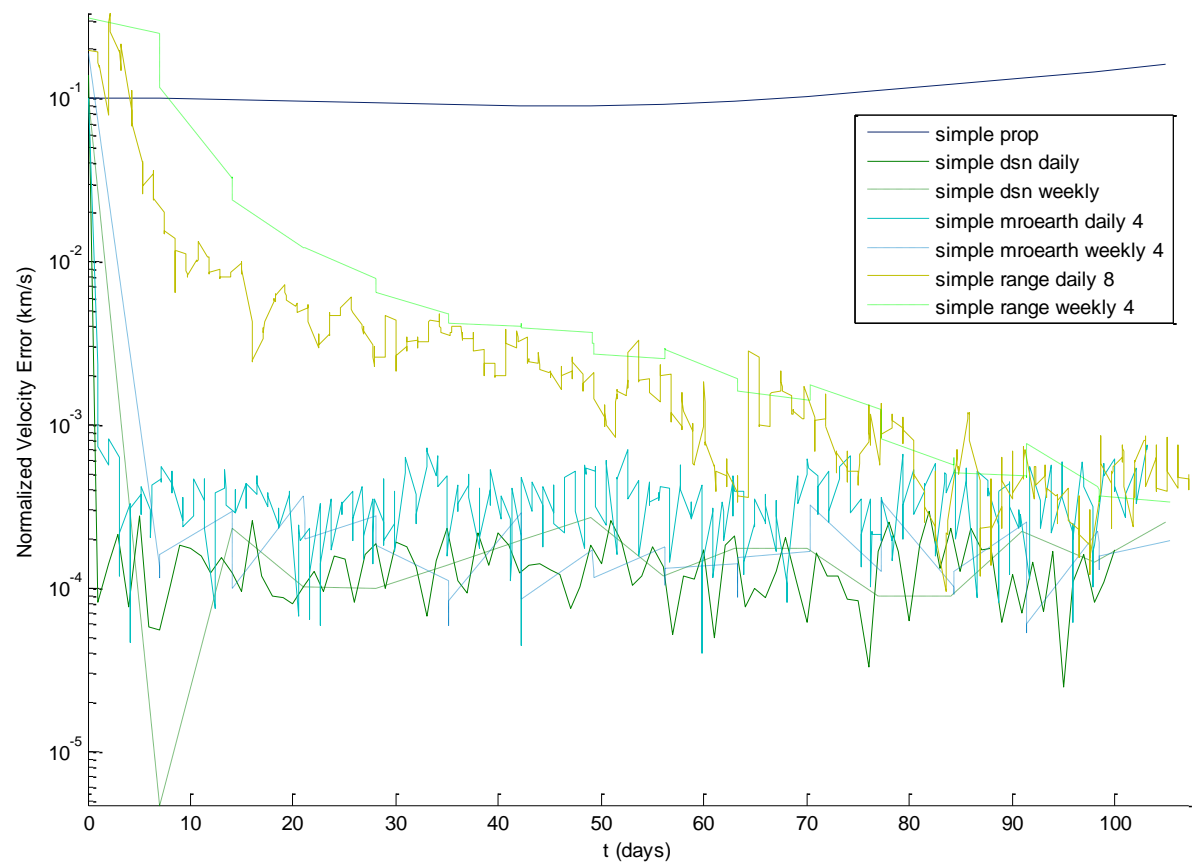

Figure 4: Velocity Error for MSL Propagated

These simulations capture the performance under an ideal, simplified dynamics model. It is important to model additional dynamical errors and perturbations in order to capture the filter's capability in a more detailed flight environment. This also captures the robustness of the estimator to flight state perturbations (such as out gassing, solar radiation pressure, or higher order gravitational effects). The filter's options can be tweaked to account for 
these uncertainties and errors in the state propagation. For these cases, in order to achieve better performance, the number of measurements for ranging on a weekly cycle was increased to 8 . The effects can be seen below in Figures 5 and 6 . The results are similar to that obtained for the simple propagation case, showing the capability of the estimator with unmodeled forces. Each case has similar, although slightly greater, estimation errors on the same order of magnitude as previously. A large difference is in the performance of the pure ranging measurements. The position errors are larger and the estimator is not able to correct over shorter timescales (thus the smoother curve), and each measurement is having a smaller effect on the correction. This is clearly seen in Figure 6 when looking at the velocity estimation errors, as the ranging case takes much longer to achieve better velocity estimation, which also impairs the ability to correctly propagate the estimated position accurately.

It is also interesting to compare the covariance in regards to the error for the various cases. It is particularly increasing when comparing the DSN measurements with the Earth and MRO-relative position measurements. These can be shown and compared in Figure 7. Each plot shows the position error of the estimator as well as 3 standard deviation bonds calculated from the filter covariance. With measurement of the complete state, it can be shown that the errors and variance come to a steady state of several kilometers, driven by the measurement error. Due to the increased error in state knowledge, the variance of the estimator with position only observations increase several orders of magnitude between measurements. This is due to the large errors present, and their effect during the long propagations between observations.

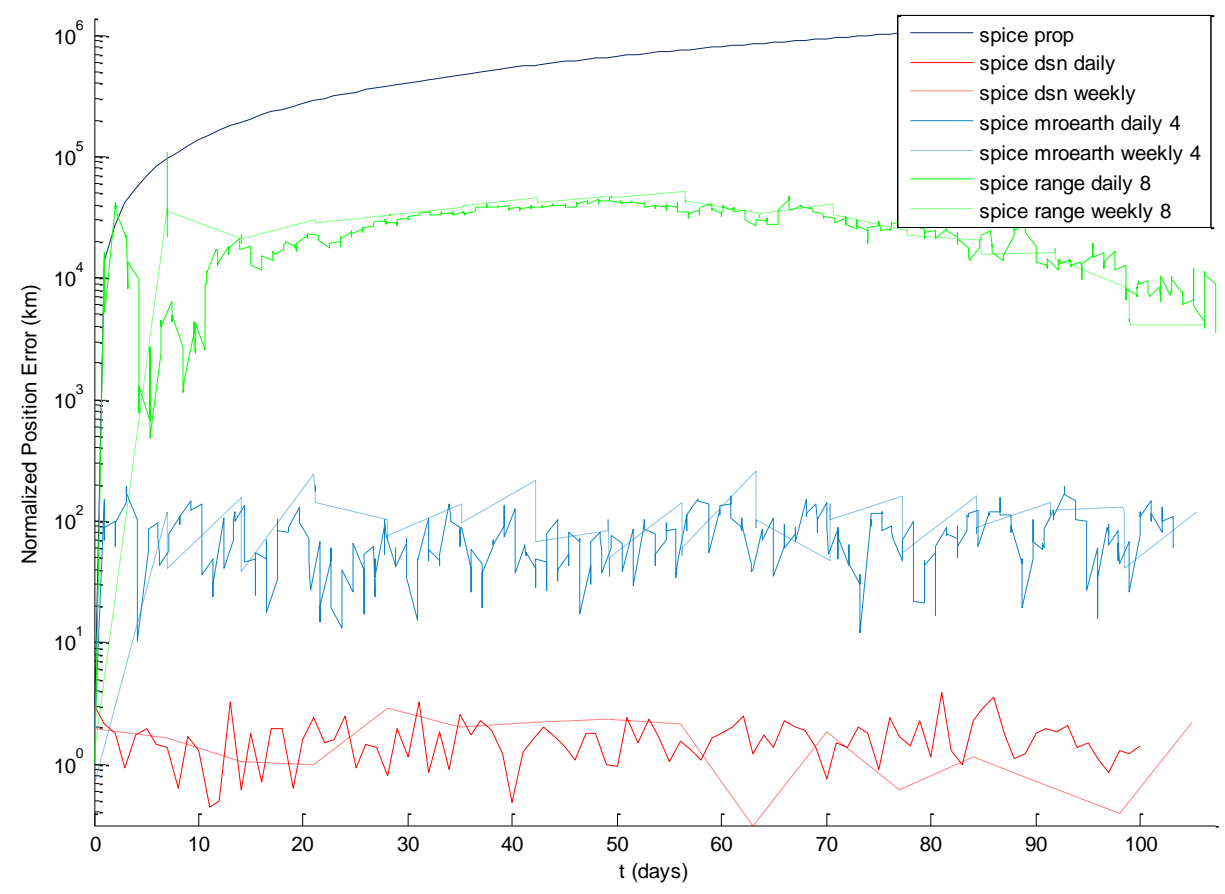

Figure 5: Position Error using SPICE Trajectory 


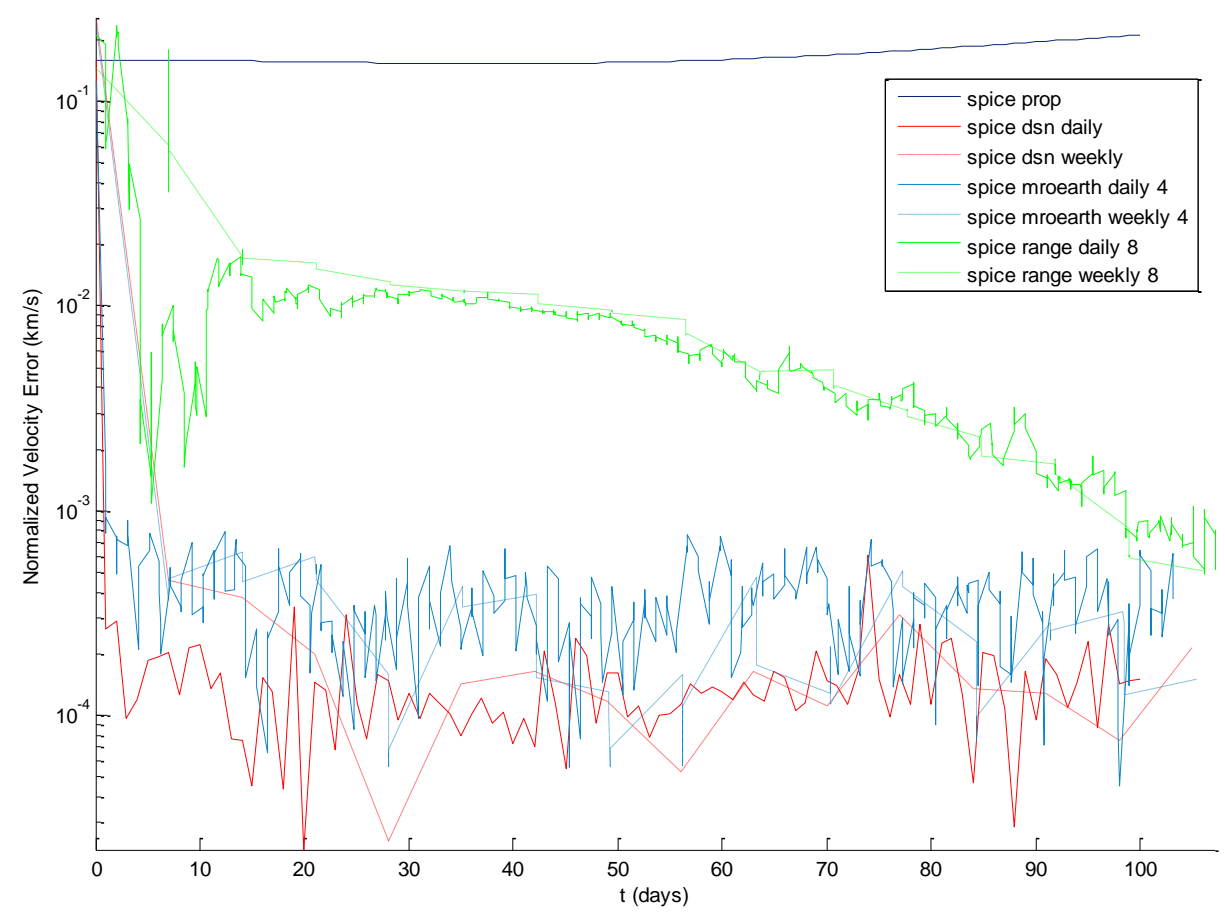

Figure 6: Velocity Error using SPICE Trajectory
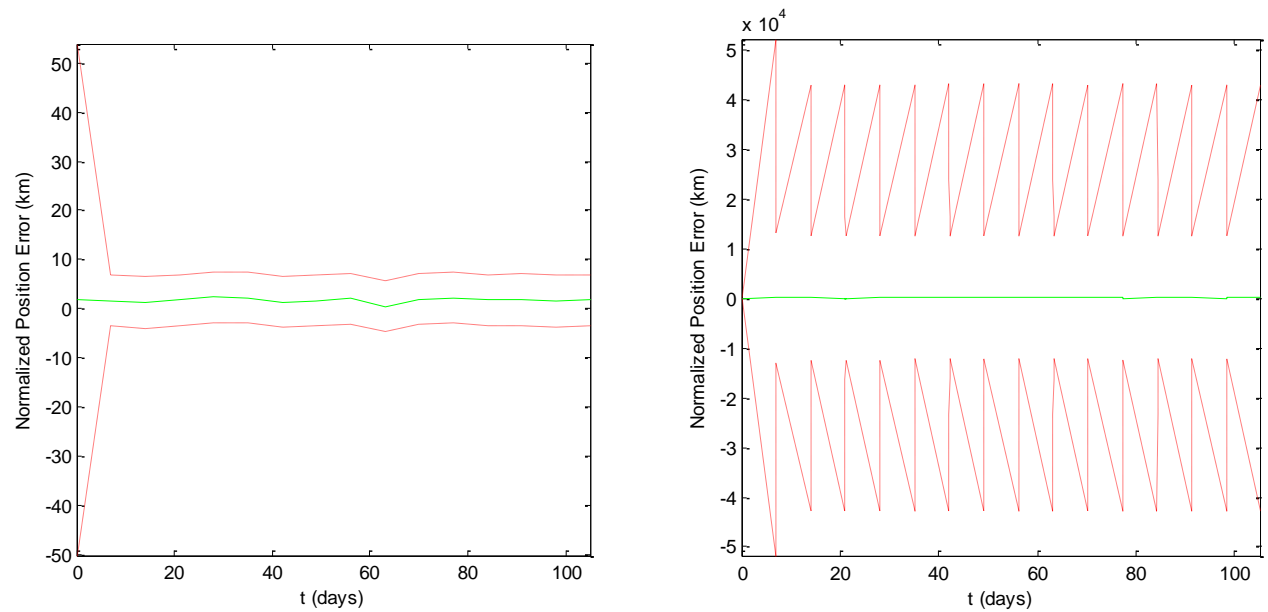

Figure 7: Error and 3 Sigma Bounds for DSN (left) and MRO/Earth Position (right)

\section{Future Work}

The research mentioned here is ongoing as of this writing, with additional formal results forthcoming. The main limitations to this analysis are the lack of disturbing forces to the spacecraft propagation models, as well as relatively simply spacecraft intelligence. Future studies will transition the analysis to a more robust simulation environment with each spacecraft operating independently, moving away from a direct integration of spacecraft state with measurements at specified intervals. Additional work is being done (and currently being implemented) on integrated communication models to capture spacecraft transmission and reception capabilities to provide a more detailed link analysis similar to Lightsey et al. ${ }^{12}$ and Munoz $^{24}$. More advanced filtering methods are also under consideration to improve the state estimation accuracy (to take into account the inherent measurement and propagation errors), such 
as Unscented Kalman Filter and Particle Filter approaches. Lastly, more detailed hardware clock models and actual hardware in the loop will be introduced to improve the fidelity of the clock modeling process.

\section{Conclusions}

As shown by the initial simulation results, the state estimation errors based on position measurements provide errors only an order of magnitude greater than current state of the art, with improved dynamical modeling and filtering techniques, there is the potential for additional improvement. Further analysis and development of spacecraft autonomy and measurement scheduling will also aid in its performance. Ranging only based on one asset though does not look achievable to support state updates, due to the large errors in the simulation. As the space network grows this could become more preferable due to ability to range off of multiple assets at once and thus reduce errors. Additionally, one limitation of this study is the focus on one type of measurement (or fixed set of) for each batch. In order to benchmark the performance in augmentation approach, it is necessary to build the modeling environment to allow for mixed packets and measurements over the course of a mission.

\section{Acknowledgments}

A NASA MSFC Office of Chief Technologist Center Innovation Fund award generously supported this work for FY2012. The authors would additionally like to acknowledge the division and branch management who continue to support this research.

\section{References}

${ }^{1}$ Curkendall, D.W., "Navigation System Design for the Mariner Jupiter/Saturn Mission," AIAA Guidance and Control Conference, AIAA, Washington, D.C. 1973. AIAA-1973-838-857..

${ }^{2}$ Riedel, J. , et al., AutoNav Mark3: Engineering the Next Generation of Autonomous Onboard Navigation and Guidance, Jet Propulsion Laboratory - California Institute of Technology, 2006, Technical Report.

${ }^{3}$ Thronton, C.L., and Border, J.S., Radiometric Tracking Techniques for Deep Space Navigation, Jet Propulsion Laboratory, California Institute of Technology, 2000.

${ }^{4}$ Hanson, J.E. , "Principles of X-Ray Navigation," Ph.D. Dissertation, Stanford University, 1986.

${ }^{5}$ Sheikh, S.I., "The Use of Variable Celestial X-Ray Sources for Spacecraft Navigation," Ph.D. Dissertation, University of Maryland, 2005.

${ }^{6}$ Sheikh, S.L., Pines, D.J. et al., "Spacecraft Navigation Using X-Ray Pulsars," Journal of Guidance, Control, and Dynamics [online journal], Vol. 29, No 1, AIAA,Washington, D.C, 2006.

${ }^{7}$ Hemmati, H. , Deep Space Optical Communications, California Institute of Technology, 2006.

${ }^{8}$ Biswas, A., and Piazzolla, S., "IPN Progress Report 42-154: Deep-Space Optical Communications Downlink Budget from Mars: System Parameters,” NASA/Jet Propulsion Laboratory, August 2003.

${ }^{9}$ Steitz, D.E., "Communications, Navigation And In-Space Propulsion Technologies Selected For NASA Flight Demonstration," URL: http://www.nasa.gov/home/hqnews/2011/aug/HQ\_11--272\_TDM\Selections.html, Release: 11-272, August 2011.

${ }^{10}$ Cerf, V. et al., "Delay-Tolerant Network Architecture: The Evolving Interplanetary Internet," Interplanetary Network Research Group, Internet Research Task Force, August 2002.

${ }^{11}$ Burleigh, S. et al. "The Interplanetary Internet: A Communications Infrastructure for Mars Exploration," 53rd International Astronautical Congress, International Astronautical Federation, October 2002.

${ }^{12}$ Lightsety, E.G, Mogensen, A.E. et al. "Real-Time Navigation for Mars Missions Using the Mars Network," Journal of Spacecraft and Rockets, AIAA, Washington, D.C., Vol. 45, No. 3, May-June, 2008.

${ }^{13}$ Scier, J.S., Rush, J.J., Williams, W.D., and Vrotsos, P., "Space Communication Architecture Supporting Exploration and Science: Plans and Studies for 2010-2030," 1st Space Exploration Conference, AIAA, Washginton, D.C., January 2005, AIAA2005-2517.

${ }^{14}$ Burleigh, S. et al., "InterPlaNetary Internet", Presentation, URL: http://www.ipnsig.org/reports/IPN-04Mar03-IPNSIG.pdf, March 2003.

${ }^{15}$ Farrel, S., and Cahill, V., Delay- and Disruption-Tolerant Networking, Artech House, Boston, 2006.

${ }^{16}$ Jones, R.M., "Deep Space Networking Experiments on the EPOXI Spacecraft," Infotech@Aerospace 2011, AIAA, Washington, D.C. March 2011. AIAA-2011-1644.

${ }^{17}$ Schoolcraft, J.B., "The Deep Impact Network Experiment - Concept, Motivation, and Results", SpaceOps 2010 Conference , AIAA, Washington, D.C, April 2010.

${ }^{18}$ Ivancic, W., "Experience with delay-tolerant networking from orbit," Advanced Satellite Mobile Systems Conference, 2008.

12

American Institute of Aeronautics and Astronautics 
${ }^{19}$ Acton, C.H.; "Ancillary Data Services of NASA's Navigation and Ancillary Information Facility;" Planetary and Space Science, Vol. 44, No. 1, pp. 65-70, 1996. =

${ }^{20}$ Axelrad, P., and Brown, R.G., "GPS Navigation Algorithms," Global Positioning System: Theory and Applications Volume 1, edited by B.W. Parkinson and J.J. Spiker, Jr., Progress in Astronautics and Aeronautics, AIAA, Washington, D.C., 1996, Ch. 9.

${ }^{21}$ Brown, R.G., and Hwang, P.Y.C., Introduction to Random Signals and Applied Kalman Filtering, 2nd ed., Wiley, New York, 1992.

${ }^{22}$ Van Dierendonck, A.J., and McGraw, J.B., "Relationship Between Allan Variances and Kalman Filter Parameters", Proceedings of the $16^{\text {th }}$ Annual Precise Time and Time Interval Systems and Applications, NASA Goddard Space Flight Center, November 1984, pp. 273-293.

${ }^{23}$ Fehlberg, E., "Classical Fifth-, Sixth-, Seventh-, and Eighth-Order Runge-Kutta Formulas with Stepsize Control", NASA TR-287, October 1968.

${ }^{24}$ Munoz, S., Christian, J.A., and Lightsey, E.G., "Development of an End to End Simulation Tool for Autonomous Cislunar Navigation," Guidance, Navigation, and Control Conference, AIAA, Washington, D.C., August, 2009. AIAA-2009-5995. 
NASA

Conceptual Design of a Communication-Based Deep Space Navigation Network

Abstract: AS the need grows for increased autonomy and position knowledge accuracy to support missions beyond Earth orbit, engineers must push and develop more advanced navigation sensors and systems that operate independent of Earth-based analysis and processing. Several spacecraft are approaching this problem using inter-spacecraft radiometric tracking and onboard autonomous
optical navigation methods. This paper proposes an alternative implementation to aid in spacecraft position fixing. The proposed method Network-Based Navigation technique takes advantage of the communication data being sent between spacecraft and between spacecraft and ground control to embed navigation information. The navigation system uses these packets to provide navigation transmitted signal itself, this method filter to augment traditional ground-based radiometric tracking techniques. As opposed to using digital signal measuen on the embedded navigation packet headers to calculate a navigation estimate. This method is heavily dependent on clock accuracy and the initial results nce of a notional system.

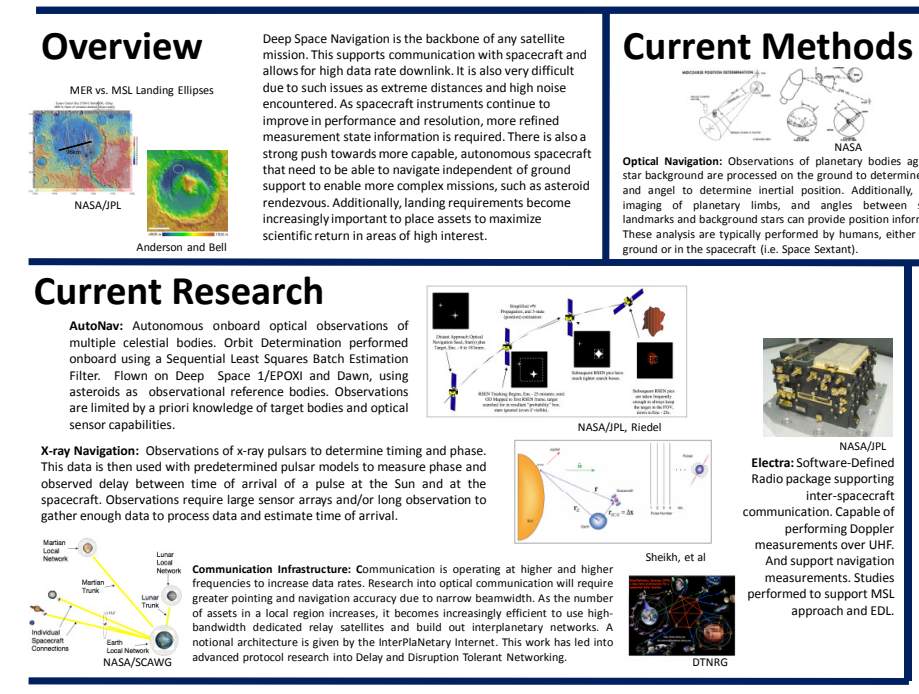

Concept of Operations

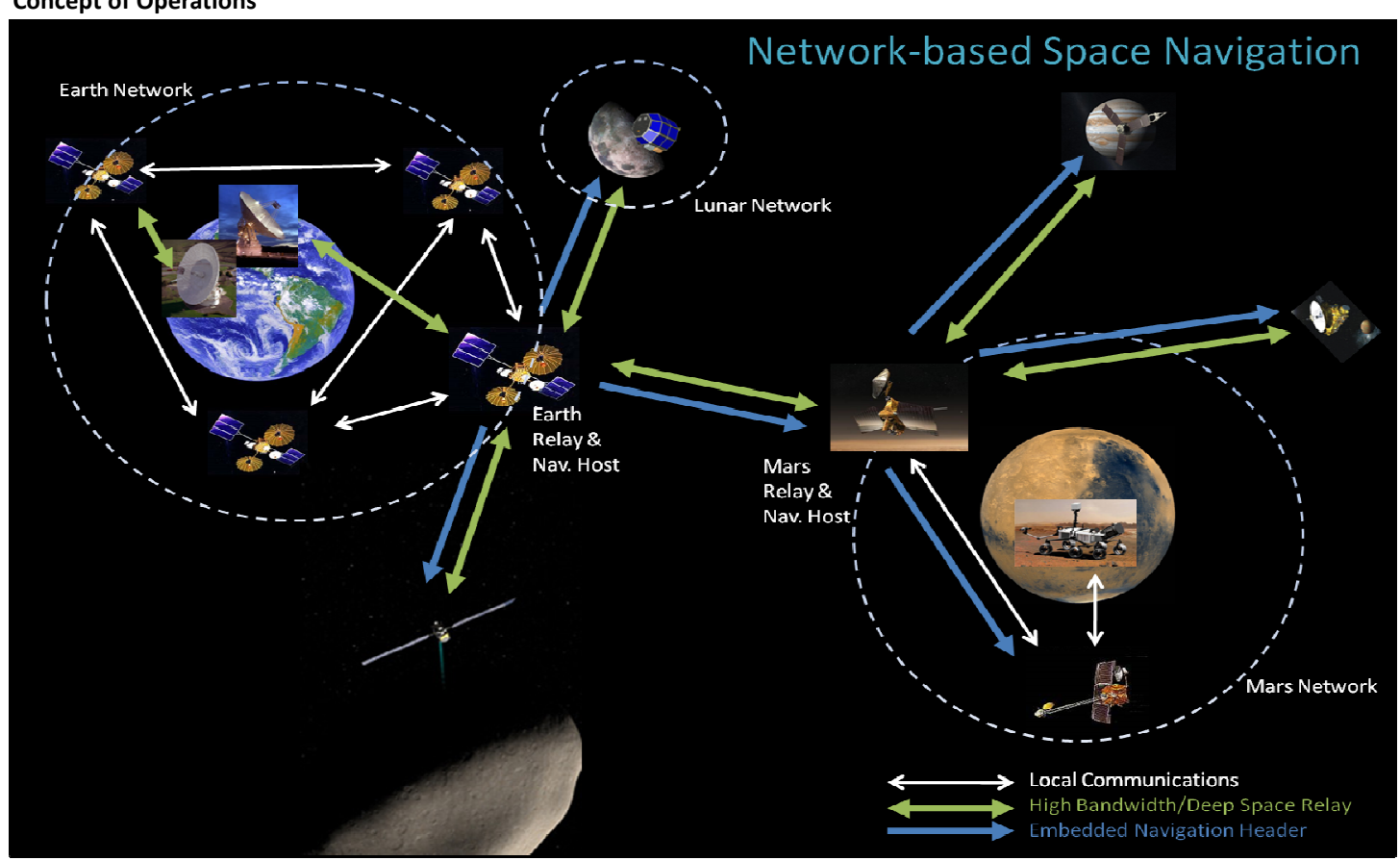

Evan J Anzalone ${ }^{1}$ and Jason C.H. Chuang ${ }^{2}$ 'Aerospace Engineer, Guidance, Navigation, and Mission Analysis Branch, MSFC, evan.j.anzalone@nasa.gov
2Navigation Team Lead, Guidance, Navigation, and Mission Analysis Branch, MSFC, jason.c.chuang@nasa.gov

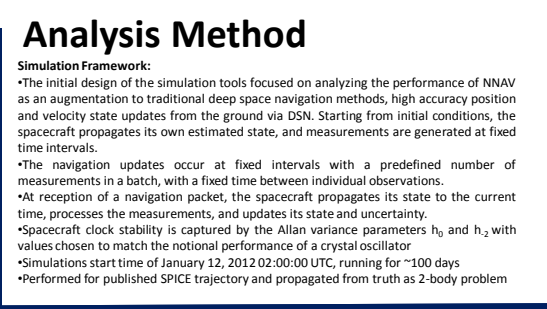

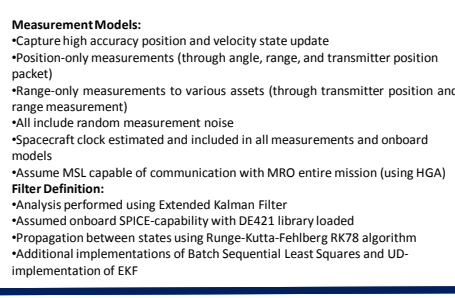

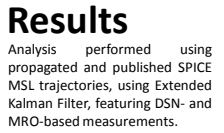
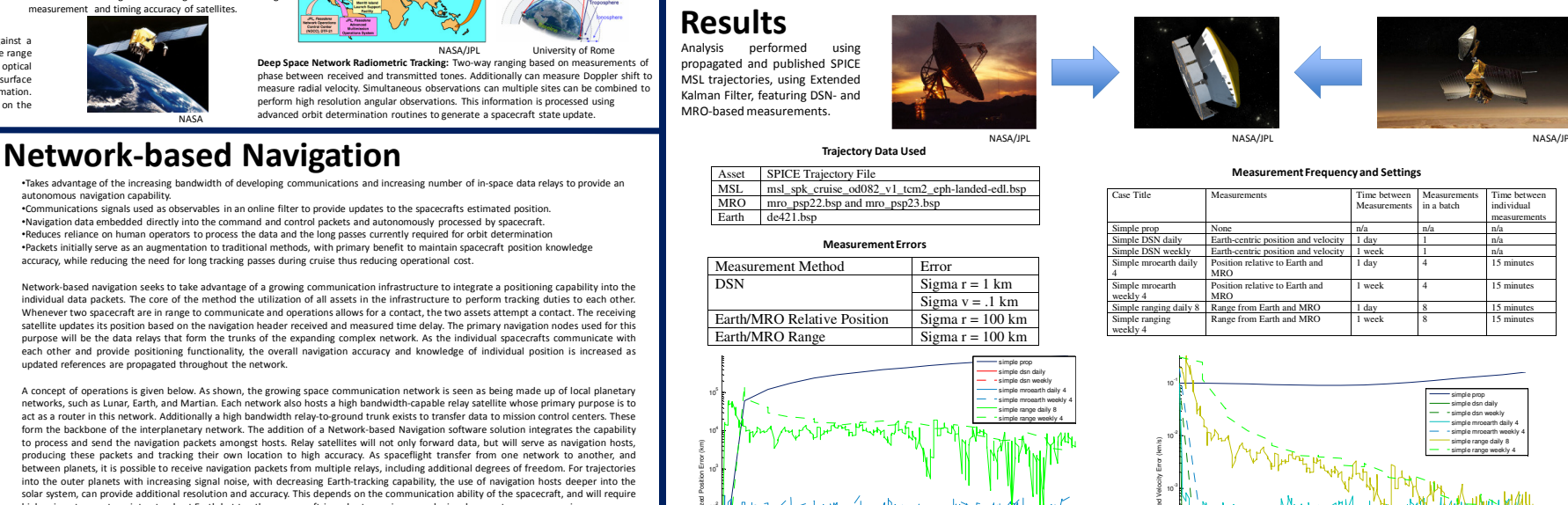

Network-based Navigation

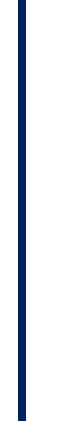

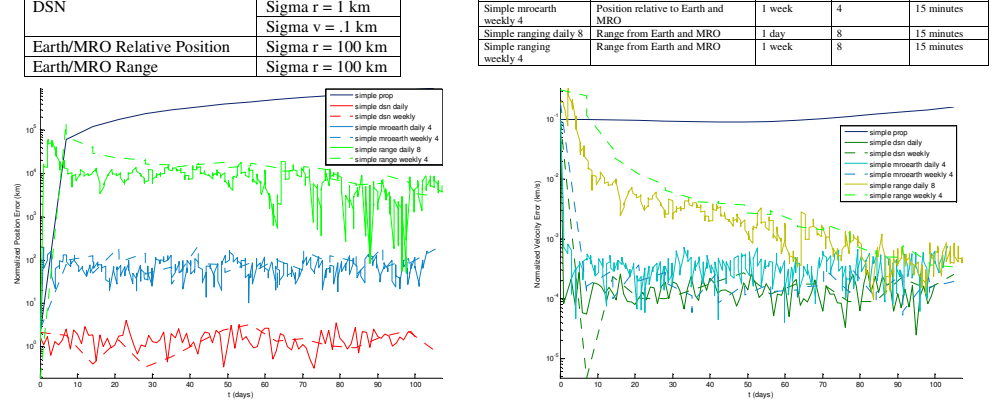

Position and Velocity Error for MSL Propagated

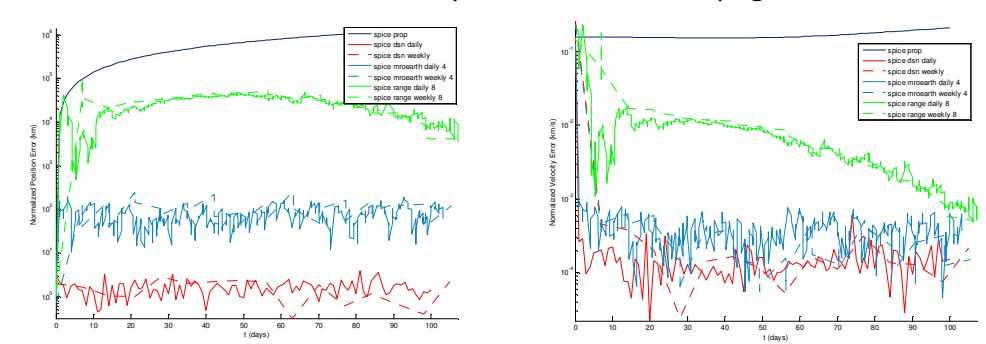

Position and Velocity Error for MSL SPICE

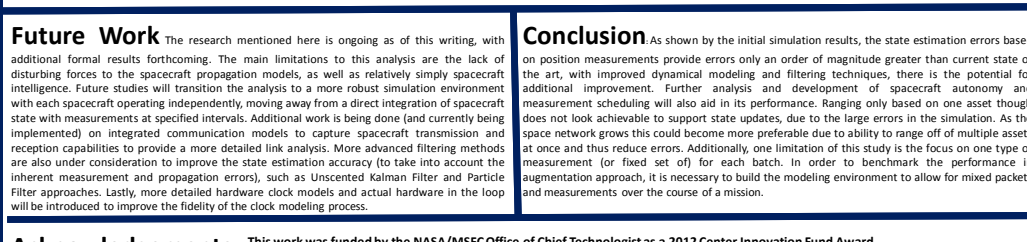

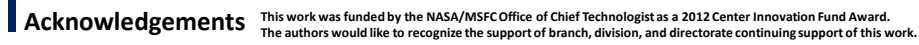

